\title{
Adaptive Neural Control of Nonaffine Nonlinear Systems without Differential Condition for Nonaffine Function
}

\author{
Chaojiao Sun, Bo Jing, and Zongcheng Liu \\ The College of Aeronautics and Astronautics Engineering, Air Force Engineering University, Xian 710038, China \\ Correspondence should be addressed to Zongcheng Liu; liu434853780@163.com
}

Received 10 December 2015; Revised 4 May 2016; Accepted 16 May 2016

Academic Editor: Haranath Kar

Copyright (C) 2016 Chaojiao Sun et al. This is an open access article distributed under the Creative Commons Attribution License, which permits unrestricted use, distribution, and reproduction in any medium, provided the original work is properly cited.

\begin{abstract}
An adaptive neural control scheme is proposed for nonaffine nonlinear system without using the implicit function theorem or mean value theorem. The differential conditions on nonaffine nonlinear functions are removed. The control-gain function is modeled with the nonaffine function probably being indifferentiable. Furthermore, only a semibounded condition for nonaffine nonlinear function is required in the proposed method, and the basic idea of invariant set theory is then constructively introduced to cope with the difficulty in the control design for nonaffine nonlinear systems. It is rigorously proved that all the closed-loop signals are bounded and the tracking error converges to a small residual set asymptotically. Finally, simulation examples are provided to demonstrate the effectiveness of the designed method.
\end{abstract}

\section{Introduction}

As a powerful technology for control design, approximationbased adaptive control has been considered extensively for uncertain nonlinear systems and attracts an ever increasing interest [1-7]. Many remarkable results have been achieved by using neural networks or fuzzy systems as universal approximators [8-16]. By employing the capability of these approximators, the unknown nonlinear functions in system can be handled with little knowledge of system plant. In particular, many important achievements have been obtained on uncertain affine nonlinear systems in which the control input appears linearly in the state equations [13-16]. However, in practice, there are many systems falling into the category featured with a cascade and nonaffine structure, such as biochemical process [17], Duffing oscillator [18], aircraft flight control system [19], and mechanical systems [20]. Moreover, it is well known that nonaffine nonlinear system has a more representative form than the affine ones, and no affine appearance of the control input to be used makes the control design more difficult [21]. Recently, some methods have been developed for nonaffine nonlinear systems with the help of the implicit function theorem [22-25]. In [22], a direct adaptive state-feedback controller is constructed for nonaffine nonlinear systems using a neural network with flexible structure. By employing Taylor series expansion of nonaffine nonlinear function, an adaptive neural control scheme is proposed with a high-gain observer in [25]. In these methods, the feasibility of using neural network to approximate the desired control input is ensured based on the implicit function theorem, and the approximation-based direct adaptive controller is then proposed for nonaffine nonlinear systems. Consequently, differential conditions for the nonaffine nonlinear functions of systems are required for the sake of using the implicit function theorem. More recently, an observer-based adaptive neural control scheme is proposed for the nonaffine nonlinear system in the presence of input saturation and external disturbance [26]. In [27], excellent control performance has been achieved by dynamic learning from adaptive neural network control for nonaffine nonlinear systems. By monitoring the tracking performance, a performance-dependent self-organizing control approach is proposed in [28].

Thus far, though many significant results have been obtained for nonaffine nonlinear systems [29-40], there are still some problems that should not be negligible, which are summarized as follows:

(1) The nonaffine nonlinear function $f(x, u)$ in (1) is assumed to be differentiable with respect to $u$ in all the 
existing methods. Apparently, this assumption is too much restrictive for applying the control methods to real systems. Moreover, as is well known, nonsmooth nonlinearities such as dead-zone and backlash exist in a wide range of real control systems [41-45], which leads to $f(x, u)$ being not partial-differentiable with respect to $u$. Consequently, the conventional control methods for nonaffine nonlinear systems will inevitably be failure. Therefore, the cancellation of differential condition for nonaffine function $f(x, u)$ has the applicable importance.

(2) Meanwhile, to ensure the control direction, the assumption that $\partial f(x, u) / \partial u$ must be strictly positive or negative is always used and viewed as the controllability condition in almost all the existing control scheme for nonaffine nonlinear systems $[25,27]$. As a result, this restrictive conditions on $\partial f(x, u) / \partial u$ severely limit the range of application of the control methods for nonaffine nonlinear systems. However, actually, the control direction can be determined even though $\partial f(x, u) / \partial u$ does not exist or $\partial f(x, u) / \partial u$ is not strictly positive or negative as shown in the later of this paper.

(3) On the other hand, many works on the controller design for nonaffine nonlinear systems are carried out with $\partial f(x, u) / \partial u$ being bounded by both upper and lower bounds [26-28]. Therefore, a priori knowledge of the plant dynamics was required to determine these bounds, which may be very difficult to acquire in practical application. The control design would be more reasonable and difficult if only semibounded condition is available. It is the above discussions that motivate our work in this paper.

The main novelties of our paper are as follows:

(1) An adaptive neural control scheme is proposed for nonaffine nonlinear systems without any differential conditions for $f(x, u)$, which suggests that the nonaffine nonlinear function $f(x, u)$ is continuous and not necessarily differentiable in our approach. It is noticeable that our method is certainly suitable for the case of $f(x, u)$ being differentiable with respect to $u$. Furthermore, the control-gain function is molded without using the mean value theorem. Hence, the control direction is ensured without any knowledge of $\partial f(x, u) / \partial u$, which is clearly different from any other methods.

(2) Compared with most of the available researches, only a semibounded condition for nonaffine nonlinear function is required in this paper, which makes the control design much more difficult. To cope with this difficulty, the basic idea of invariant set theory is constructively introduced in this control design for nonaffine nonlinear systems in the light of [46]. This enables the proposed scheme to have great potential in practical application.
(3) The number of the online adaptive parameter is only two and is independent of the dimensionality of system. Furthermore, semiglobal uniform boundedness stability is rigorously established using the Lyapunov approach.

The rest of this paper is organized as follows. Section 2 gives the problem formulation and preliminaries. Adaptive neural controller is developed for a class of nonaffine nonlinear system with external disturbance in Section 3. The stability analysis of the closed-loop system is given in Section 4 using Lyapunov analysis theory and invariant set theory. In Section 5, simulation studies are performed to show the effectiveness of the proposed scheme. Finally, the conclusion is included in Section 6.

\section{Problem Statement and Preliminaries}

2.1. Problem Formulation. Consider a class of uncertain SISO nonaffine nonlinear system as follows:

$$
\begin{aligned}
\dot{x}_{i} & =x_{i+1}, \quad i=1,2 \ldots, n-1, \\
\dot{x}_{n} & =f(x, u)+d(t), \\
y & =x_{1},
\end{aligned}
$$

where $x=\left[x_{1}, x_{2}, \ldots, x_{n}\right]^{T} \in R^{n}$ denotes the state vector of the system; $u \in R$ and $y \in R$ are the control input and system output, respectively. The unknown continuous function $f(x, u)$ represents nonlinearities that are nonaffine for the control signal $u$, and $d(t)$ represents the external disturbance of system. It is noted that the nonaffine function $f(x, u)$ is assumed to be continuous rather than smooth or differentiable in our paper.

The control objective is to design adaptive neural tracking control $u$ such that the system output $y$ follows the desired trajectory $y_{d}$ in the presence of external disturbance and nonaffine nonlinearity.

The main difficulty of this control design problem is that the system input $u$ does not appear linearly, which makes the direct feedback linearization difficult or impossible. Define the function

$$
F(x, u)=f(x, u)-f(x, 0) .
$$

Before proceeding to the direct adaptive neural control design of system (1), let us consider the following assumptions.

Assumption 1. For all $x$ and $u$ in system (1), there exist constants $m, m^{\prime}, c$, and $c^{\prime}$ such that

$$
\begin{aligned}
& F(x, u) \geq m u+c, \quad u \geq 0, \\
& F(x, u) \leq m^{\prime} u+c^{\prime}, \quad u<0,
\end{aligned}
$$

where $m$ and $m^{\prime}$ are positive constants.

Remark 2. It should be noticed that nonaffine function $F(x, u)$ is continuous and not necessarily differentiable in Assumption 1 . Therefore, there is no differential condition for $f(x, u)$ in our method. This fact distinguishes our proposed control system from all the existing methods. 
Remark 3. It is worth mentioning that $F(x, u)$ is semibounded in Assumption 1; namely, the upper bound and lower bound of $F(x, u)$ in the case of $u \geq 0$ and $u<0$ are cancelled, respectively. This is also quite different from other studies and makes the design work much more challenge in our paper. The merits for the cancellation of those bounds are illustrated as follows:

(1) Consider the nonaffine nonlinear function $f(x, u)=$ $x_{1}^{2}+0.15 u^{3}+0.1\left(1+x_{2}^{2}\right) u+\sin (0.1 u)$ in simulation example of [22] in which the assumption of $0<$ $\partial f(x, u) / \partial u<c$ is required with the constant $c$ being design parameter. However, it is doubtful that whether the inequality $\partial f(x, u) / \partial u<c$ will be always satisfied in the simulation of [22] because the design parameter $c$ must be specified by designer previously and $\partial f(x, u) / \partial u$ is time-varying. Therefore, this problem makes the simulation in [22] questionable. However, it is easily known that $f(x, u)-f(x, 0) \geq$ $0.1 u-1$ for $u \geq 0$ and $f(x, u)-f(x, 0) \leq 0.1 u+1$ for $u<0$. Hence, our method is suitable for this example without doubt.

(2) In [26, 27], the adaptive tracking controller was constructed under the assumption that $0<\varepsilon \leq$ $\partial f(x, u) / \partial u<c$ with $\varepsilon$ and $c$ being positive constant or positive-defined function of $x$. By integrating $0<$ $\varepsilon \leq \partial f(x, u) / \partial u<c$ with respect to $u$ over $[0, u]$, we can easily obtain

$$
\begin{array}{ll}
\varepsilon u \leq f(x, u)-f(x, 0) \leq c u, \quad u \geq 0, \\
\varepsilon u \geq f(x, u)-f(x, 0) \geq c u, \quad u<0
\end{array}
$$

which can be rewritten as

$$
\begin{array}{ll}
\varepsilon u \leq F(x, u) \leq c u, & u \geq 0, \\
\varepsilon \mathcal{E} \geq F(x, u) \geq c u, \quad u<0 .
\end{array}
$$

Comparing the above inequalities with (3) of Assumption 1, it can be found that Assumption 1 is apparently more relaxed.

Assumption 4. For all $t>0$, there exist unknown positive constant $d^{*}$ such that $|d(t)| \leq d^{*}$.

Assumption 5. Define the desired trajectory as $y_{d}$; we assume that the reference signals $y_{d}, \dot{y}_{d}, \ddot{y}_{d}, \ldots, y_{d}^{(n)}$ are smooth and bounded; that is, there exists a positive constant $K_{0}$ such that $\Pi_{0}:=\left\{\left(y_{d}, \dot{y}_{d}, \ldots, y_{d}^{(n)}\right): \sum_{i=0}^{n}\left(y_{d}^{(i)}\right)^{2} \leq K_{0}\right\}$, where $y_{d}^{(0)}$ denotes $y_{d}$. For conciseness, define $x_{d}=\left[y_{d}, \dot{y}_{d}, \ldots, y_{d}^{(n)}\right]$ throughout this paper.

Lemma 6 (see [47]). Consider the dynamic system in the form of

$$
\dot{\chi}(t)=-a \chi(t)+\xi v(t)
$$

where $a$ and $\xi$ are positive constants and $v(t)$ is a positive function. Then, for any given bounded initial condition $\chi(0) \geq$ 0 , we have $\chi(t) \geq 0, \forall t \geq 0$.
Lemma 7 (see [6]). Hyperbolic tangent function tanh(·) will be used in this paper, and it is well known that $\tanh (\cdot)$ is continuous and differentiable, and it fulfils that for any $q \in R$ and $\forall v>0$

$$
\begin{aligned}
& 0 \leq|q|-q \tanh \left(\frac{q}{v}\right) \leq 0.2785 v, \\
& 0 \leq q \tanh \left(\frac{q}{v}\right)
\end{aligned}
$$

Lemma 8. For $\forall a, b \in R$, if $a \leq x \leq b$, then $x=\theta a+(1-\theta) b$, where $\theta=(b-x) /(b-a)$.

Proof. Substituting $\theta=(b-x) /(b-a)$ into the term $\theta a+(1-$ $\theta) b$, then we will find that $x=\theta a+(1-\theta) b$ is an identical equation. And it is easy to know that $0 \leq \theta \leq 1$ by noting $a \leq x \leq b$.

2.2. RBFNN Basics. The radial basis function neural network (RBFNN) is considered to be used for the controller design in this paper, which is utilized to approximate the continuous function $h(Z): R^{n} \rightarrow R$

$$
h_{n n}(Z)=W^{T} \phi(Z) \text {, }
$$

where the input vector $Z \in \Omega_{Z} \subset R^{n}$, weights vector $W=$ $\left[W_{1}, W_{2}, \ldots, W_{l}\right] \in R^{l}$, the neural network node number $l>$ 1 , and $\phi(Z)=\left[\phi_{1}(Z), \ldots, \phi_{l}(Z)\right]^{T}$ with $\phi_{i}(Z)$ being chosen as the commonly used Gaussian functions as

$$
\phi_{i}(Z)=\exp \left[\frac{-\left(Z-\mu_{i}\right)^{T}\left(Z-\mu_{i}\right)}{\eta^{2}}\right], \quad i=1,2, \ldots, l,
$$

where $\mu_{i}=\left[\mu_{i 1}, \mu_{i 2}, \ldots, \mu_{i n}\right]^{T}$ is the center of the respective field and $\eta$ is the width of the Gaussian function.

It has been proven that network (8) can approximate any continuous function over a compact set $\Omega_{Z} \subset R^{n}$ to any desired accuracy in the form of

$$
h(Z)=W^{* T} \phi(Z)+\varepsilon(Z), \quad \forall Z \in \Omega_{Z} \subset R^{n},
$$

where $W^{*}$ is the ideal constant weight vector and $\varepsilon(Z)$ is the approximation error which is bounded over the compact set; that is, $\|\varepsilon(Z)\| \leq \varepsilon^{*}, \forall Z \in \Omega_{Z}$ with $\varepsilon^{*}>0$ being an unknown constant. In this paper, $\varepsilon(Z)$ is denoted as $\varepsilon$ to simplify the notation.

The optimal weight vector $W^{*}$ is an "artificial" quantity required only for analytical purposes. Typically, $W^{*}$ is chosen as the value of $W$ that minimizes $\varepsilon$ over $\Omega_{Z}$; that is,

$$
W^{*}:=\arg \min _{W \in R^{l}}\left\{\sup _{Z \in \Omega_{Z}}\left|h(Z)-W^{T} \phi(Z)\right|\right\} .
$$

In this paper, let $\|a\|$ and $\|A\|$ denote the 2-norm of a vector $a$ and a square matrix $A$, respectively.

\section{Adaptive Neural Tracking Controller Design}

In this section, adaptive neural tracking controller will be developed for the uncertain nonaffine nonlinear system (1). 
The design work is under the condition that the full state $x$ of system (1) is available for feedback. To begin with this work, we define

$$
e=\left[e_{1}, e_{2}, \ldots, e_{n}\right]^{T}, \quad e_{i}=x_{i}-y_{d}^{(i-1)} .
$$

In accordance with (12), the filtered tracking error of nonaffine nonlinear system (1) is defined as follows:

$$
s=\left(\frac{d}{d t}+q\right)^{n-1} e_{1}=\left[\lambda_{1}, \lambda_{2}, \ldots, \lambda_{n-1}, 1\right] e,
$$

where $\lambda_{i}=C_{n-1}^{i-1} q^{n-i},(i=1, \ldots, n-1)$ and $q>0$ are positive constants, specified by designer.

Remark 9. It has been shown in [48] that definition (13) has the following properties:

(a) $s=0$ defines a time-varying hyperplane in $R^{n}$ on which the tracking error $e_{1}$ converges to zero asymptotically.

(b) If $|s(t)| \leq C, \forall t \geq 0$ with constant $C>0$, then $e(t)$ will converge to $\Omega_{c}$, which is specified as

$$
\Omega_{c}=\left\{e|| e_{i} \mid \leq 2^{i-1} q^{i-n} C, \quad i=1,2, \ldots, n\right\}, \quad \forall t \geq T_{0}
$$

with $T_{0} \geq 0$ a computable constant.

Subsequently, to confine $e_{1}$ to a small neighborhood of origin, the regulation of $s$ will be investigated in the following.

Differentiating (13) and using (1) and (12) yields

$$
\dot{s}=f(x, u)+d(t)-y_{d}^{(n)}+\sum_{i=1}^{n-1} \lambda_{i} e_{i+1} .
$$

Consider the stabilization of system (15) and the following quadratic function candidate:

$$
V_{s}=\frac{1}{2} s^{2}
$$

The time derivative of $V_{s}$ along (15) is

$$
\dot{V}_{s}=s f(x, u)+s d(t)-s y_{d}^{(n)}+s \sum_{i=1}^{n-1} \lambda_{i} e_{i+1} .
$$

Using Assumption 4 and the definition of $F(x, u)$, we have

$$
\dot{V}_{s} \leq s[F(x, u)+f(x, 0)]+|s| d^{*}+s Y_{d},
$$

where $Y_{d}=-y_{d}^{(n)}+\sum_{i=1}^{n-1} \lambda_{i} e_{i+1}$. Due to the presence of unknown continuous function $f(x, 0)$, a RBFNN is then considered to be used to approximate it as follows:

$$
f(x, 0)=W^{* T} \varphi(x)+\varepsilon, \quad \forall x \in \Omega_{x},
$$

where $\varepsilon$ is the approximation error, satisfying $|\varepsilon| \leq \varepsilon^{*}$ with $\varepsilon^{*}>0$ being an unknown constant. Using (19), we can further obtain

$$
\dot{V}_{s} \leq s F(x, u)+s W^{* T} \varphi(x)+|s| \varepsilon^{*}+|s| d^{*}+s Y_{d} .
$$

In view of Young's inequality, we have that

$$
s W^{* T} \varphi(x) \leq \frac{s^{2}\left\|W^{*}\right\|^{2}}{2 a^{2}} \varphi^{T}(x) \varphi(x)+\frac{a^{2}}{2},
$$

where $a$ is any positive constant. Substituting (21) into (20) yields

$$
\begin{aligned}
\dot{V}_{s} \leq & s F(x, u)+\frac{s^{2}\left\|W^{*}\right\|^{2}}{2 a^{2}} \varphi^{T}(x) \varphi(x)+\frac{a^{2}}{2}+|s| \varepsilon^{*} \\
& +|s| d^{*}+s Y_{d} .
\end{aligned}
$$

Consider the following Lyapunov function:

$$
V=V_{s}+\frac{m_{a}}{2 \gamma} \widetilde{\delta}^{2}+\frac{m_{a}}{2 \beta} \widetilde{\theta}^{2}
$$

where $\widetilde{\delta}=\delta-\widehat{\delta}, \widetilde{\theta}=\theta-\widehat{\theta}$, and $m_{a}$ is a positive constant and defined as $m_{a}=\min \left\{m, m^{\prime}\right\} . \widehat{\theta}$ is the estimate of $\theta$ which is defined as $\theta=m_{a}^{-1}\left\|W^{*}\right\|^{2} . \widehat{\delta}$ is the estimate of $\delta$ which is unknown constant and will be defined later. $\gamma>0$ and $\beta>0$ are design parameters.

Then, we can determine the control law and its adaption laws as follows:

$$
\begin{aligned}
u= & -k s-\frac{\widehat{\theta} s}{2 a^{2}} \varphi^{T}(x) \varphi(x)-\widehat{\delta} \tanh \left(\frac{s}{v}\right) \\
& -\zeta Y_{d} \tanh \left(\frac{s Y_{d}}{v}\right), \\
\dot{\hat{\delta}}= & \gamma s \tanh \left(\frac{s}{v}\right)-\sigma_{1} \gamma \widehat{\delta}, \\
\dot{\hat{\theta}}= & \frac{\beta s^{2}}{2 a^{2}} \varphi^{T}(x) \varphi(x)-\sigma_{2} \beta \widehat{\theta},
\end{aligned}
$$

where $k>0, a>0, v>0, \sigma_{1}>0, \sigma_{2}>0, \gamma>0, \beta>0$, and $\zeta \geq m_{a}^{-1}$ are design parameters.

Remark 10. From (12) and the definition of $s$ and $Y_{d}$, it is easily known that $x, s$, and $Y_{d}$ can be expressed in form of $e_{1}, e_{2}, \ldots, e_{n}, y_{d}, \dot{y}_{d}, \ldots, y_{d}^{(n)}$. Therefore, it can be learned from (24) that $u$ can be expressed as a continuous function in form of $\widehat{\theta}, \widehat{\delta}, e_{1}, e_{2}, \ldots, e_{n}, y_{d}, \dot{y}_{d}, \ldots, y_{d}^{(n)}$. It should be noted that $F(x, u)$ is a continuous function of $x$ and $u$. Thus, it can be concluded that $F(x, u)$ is a continuous function of $\widehat{\theta}, \widehat{\delta}, e_{1}, e_{2}, \ldots, e_{n}, y_{d}, \dot{y}_{d}, \ldots, y_{d}^{(n)}$. In the sequel, we have that $F(x, u)$ can be expressed in the following form:

$$
F(x, u)=\kappa\left(\widehat{\theta}, \widehat{\delta}, e_{1}, e_{2}, \ldots, e_{n}, y_{d}, \dot{y}_{d}, \ldots, y_{d}^{(n)}\right),
$$

where $\kappa(\cdot)$ is an introduced continuous function. This will be useful in the stability analysis hereinafter.

\section{Main Results}

In this section, the main results of this paper will be stated, and stability analysis will be given. The main results of our paper are given as follows. 
Theorem 11. Consider the closed-loop system consisting of the nonaffine nonlinear system (1) satisfying Assumptions 1, 4, and 5, the control law (24), and the adaption laws (25) and (26). Given any $p>0$, then, for bounded initial conditions satisfying $\widehat{\theta}(0) \geq 0, \widehat{\delta}(0) \geq 0$, and $V(0) \leq p$, there exist $k, a, v, \sigma_{1}, \sigma_{2}$, $\gamma, \beta$, and $\zeta$ such that $V(t) \leq p$ for $\forall t>0$ and the following properties are guaranteed:

(i) All of the closed-loop signals are bounded, and the state vector $x$ remains within

$$
\begin{array}{r}
\Omega_{x}=\left\{x(t) \mid\|e\| \leq \eta, i=1,2, \ldots, n, x_{d} \in \Pi_{0}\right\}, \\
\forall t \geq 0,
\end{array}
$$

where the size of the positive constant $\eta$ depends on the initial conditions and design parameters.

(ii) The filtered tracking error $s$ and tracking error e will eventually converge to compact sets $\Omega_{s}$ and $\Omega_{c}$, respectively, defined by

$$
\begin{aligned}
& \Omega_{s}=\{s|| s \mid \leq C\}, \\
& \Omega_{c}=\left\{e|| e_{i} \mid \leq 2^{i-1} q^{i-n} C, i=1,2, \ldots, n\right\},
\end{aligned}
$$

where $C>0$ is a constant related to the design parameters. Therefore, $\Omega_{s}$ and $\Omega_{c}$ can be made as small as desired using a trial-and-error method to obtain the appropriate design parameters.

Proof. Considering the set $\Pi_{1}:=\left\{s^{2}+m_{a} \widetilde{\delta}^{2} / \gamma+m_{a} \widetilde{\theta}^{2} / \beta \leq 2 p\right\}$ and noting that $s=\sum_{i=1}^{n-1} \lambda_{i} e_{i}+e_{n}$, we have $\Pi_{1}=\left\{\left(\lambda_{1} e_{1}+\lambda_{2} e_{2}+\right.\right.$ $\left.\left.\lambda_{3} e_{3}+\cdots+e_{n}\right)^{2}+m_{a} \widetilde{\delta}^{2} / \gamma+m_{a} \widetilde{\theta}^{2} / \beta \leq 2 p\right\}$ with polynomial $s^{n-1}+\lambda_{n-1} s^{n-2}+\cdots+\lambda_{1}$ being Hurwitz. Consequently, we can conclude that $\Pi^{\prime}=\Pi_{0} \times \Pi_{1} \in R^{2 n+3}$ is compact because $\Pi_{0} \in R^{n+1}$ and $\Pi_{1} \in R^{n+2}$ are compact. It is easy to see from (27) that all variables of the continuous function $\kappa(\cdot)$ are in the compact $\Pi^{\prime}$. Therefore, $|\kappa(\cdot)|$ has a maximum $M$ on $\Pi_{0} \times \Pi_{1}$, where $M>0$ is an unknown constant. Noting (27), we have that, on $\Pi_{0} \times \Pi_{1}$, the following inequality is satisfied:

$$
|F(x, u)| \leq M
$$

which can be rewritten as

$$
-M \leq F(x, u) \leq M .
$$

Similarly, there exists an unknown positive constant $L$ such that

$$
|F(x, 0)| \leq L
$$

on the compact set $\Pi_{0} \times \Pi_{1}$.

Remark 12. It should be mentioned that (30) and (31) do not contradict with Assumption 1. Equation (30) is satisfied only for $\forall(x, u) \in \Pi_{0} \times \Pi_{1}$, while Assumption 1 is imposed for all $x$ and $u$. From Remark 10, we have $F(x, 0)=$ $\kappa_{0}\left(e_{1}, e_{2}, \ldots, e_{n}, y_{d}, \dot{y}_{d}, \ldots, y_{d}^{(n)}\right)$ with $\kappa_{0}(\cdot)$ being a continuous function; therefore, (32) is satisfied on the set $\Pi_{0} \times \Pi_{1}$. The following analysis are based on (30)-(32); thus, we need to prove that all the variables of $\Pi_{1}$ will stay in it. Note that $V(0) \leq p$, which implies that all the initial conditions of the concerned variables are within $\Pi_{1}$. Therefore, in the sequel, we only need to prove that $\Pi_{1}$ is an invariant set.

Using (3) and (31), one obtain

$$
\begin{aligned}
m u+c & \leq F(x, u) \leq M, \quad u \geq 0, \\
-M & \leq F(x, u) \leq m^{\prime} u+c^{\prime}, \quad u<0
\end{aligned}
$$

which can be further rewritten as

$$
\begin{array}{r}
m u+c \leq F(x, u) \leq \frac{M-c}{u} u+c, \quad u>0, \\
\frac{-M-c^{\prime}}{u} u+c^{\prime} \leq F(x, u) \leq m^{\prime} u+c^{\prime}, \quad u<0 .
\end{array}
$$

Define $g_{1}(u)=(M-c) / u$ and $g_{2}(u)=\left(-M-c^{\prime}\right) / u$. Then we can know from (34) that $g_{1}(u) \geq m$ for $\forall u>0$ and $g_{2}(u) \geq m^{\prime}$ for $\forall u<0$. We can further rewrite (34) as

$$
\begin{aligned}
m u+c & \leq F(x, u) \leq g_{1}(u) \cdot u+c, \quad u>0, \\
g_{2}(u) \cdot u+c^{\prime} & \leq F(x, u) \leq m^{\prime} u+c^{\prime}, \quad u<0 .
\end{aligned}
$$

Based on Lemma 8, it can be learned from (35) that there exist functions $\mu_{1}(t)$ and $\mu_{2}(t)$ taking values in the closed interval $[0,1]$ and satisfying

$$
\begin{aligned}
F(x, u)= & \mu_{1}(t)(m u+c) \\
& +\left(1-\mu_{1}(t)\right)\left(g_{1}(u) \cdot u+c\right), \quad u>0, \\
F(x, u)= & \mu_{2}(t)\left(g_{2}(u) \cdot u+c^{\prime}\right) \\
& +\left(1-\mu_{2}(t)\right)\left(m^{\prime} u+c^{\prime}\right), \quad u<0
\end{aligned}
$$

which can be further rewritten as

$$
\begin{aligned}
F(x, u)=\mu_{1}(t) m u+\left(1-\mu_{1}(t)\right) g_{1}(u) \cdot u+c, & \\
u & >0, \\
F(x, u)=\mu_{2}(t) g_{2}(u) \cdot u+\left(1-\mu_{2}(t)\right) m^{\prime} u+c^{\prime}, & \\
u & <0 .
\end{aligned}
$$

To facilitate the control design, define piecewise functions $g(t)$ and $\Delta(t)$ as

$$
\begin{aligned}
& g(t)= \begin{cases}\mu_{1}(t) m+\left(1-\mu_{1}(t)\right) g_{1}(u), & u>0, \\
m, & u=0, \\
\mu_{2}(t) g_{2}(u)+\left(1-\mu_{2}(t)\right) m^{\prime}, & u<0,\end{cases} \\
& \Delta(t)= \begin{cases}c, & u>0, \\
F(x, u), & u=0, \\
c^{\prime}, & u<0 .\end{cases}
\end{aligned}
$$


Then, we can know that

$$
\begin{aligned}
& 0<m_{a} \leq g(t), \\
& 0<|\Delta(t)| \leq \Delta^{*},
\end{aligned}
$$

where $m_{a}$ is defined in (23) and $\Delta^{*}=\max \left\{L,|c|,\left|c^{\prime}\right|\right\}$. With the help of (38), we can rewrite (37) as

$$
F(x, u)=g(t) u+\Delta(t)
$$

Then, noting the definition of $F(x, u)$, the original system (1) can be rewritten as

$$
\begin{aligned}
\dot{x}_{i} & =x_{i+1}, \quad i=1,2 \ldots, n-1, \\
\dot{x}_{n} & =g(t) u+\Delta(t)+f(x, 0)+d(t), \\
y & =x_{1} .
\end{aligned}
$$

Remark 13. It can be seen from (39) and (41) that the control direction is positive in our method, which guarantees the controllability of system. And the control-gain function $g(t)$ is obtained without any prior knowledge of $\partial f(x, u) / \partial u$, which implies that the conditions of $\partial f(x, u) / \partial u$ are no longer required to obtain the control direction. This is quite different from other researches. To further show that the control direction is independent of the condition of $\partial f(x, u) / \partial u$ being strictly positive or negative, let us consider the function $f(x, u)=\left(1+0.1 \cos x_{1}\right)(1+0.2 \cos u) u$. It is easily known that $\partial f(x, u) / \partial u$ is positive at $u=\pi / 2$ and negative at $u=5 \pi / 2$. So it is not strictly positive or negative. But it still satisfies Assumption 1 because $f(x, u)-f(x, 0) \geq 0.72 u$ for $u \geq 0$ and $f(x, u)-f(x, 0) \leq 0.72 u$ for $u<0$, and hence it follows from the analysis of this paper that the control direction is positive in the case of $f(x, u)=\left(1+0.1 \cos x_{1}\right)(1+0.2 \cos u) u$.

Noticing (22), (23), and (40), it can be known from the previous analysis that the time derivative of $V$ is

$$
\begin{aligned}
\dot{V} \leq & s g(t) u+s \Delta(t)+\frac{s^{2}\left\|W^{*}\right\|^{2}}{2 a^{2}} \varphi^{T}(x) \varphi(x)+\frac{a^{2}}{2} \\
& +|s| \varepsilon^{*}+|s| d^{*}+s Y_{d}+\frac{m_{a}}{\gamma} \widetilde{\delta} \dot{\tilde{\delta}}+\frac{m_{a}}{\beta} \tilde{\theta} \dot{\tilde{\theta}} \\
\leq & s g(t) u+|s| \Delta^{*}+\frac{s^{2}\left\|W^{*}\right\|^{2}}{2 a^{2}} \varphi^{T}(x) \varphi(x)+\frac{a^{2}}{2} \\
& +|s| \varepsilon^{*}+|s| d^{*}+s Y_{d}-\frac{m_{a}}{\gamma} \widetilde{\delta} \dot{\hat{\delta}}-\frac{m_{a}}{\beta} \tilde{\theta} \dot{\hat{\theta}} \\
\leq & s g(t) u+\frac{s^{2}\left\|W^{*}\right\|^{2}}{2 a^{2}} \varphi^{T}(x) \varphi(x)+\frac{a^{2}}{2}+|s| m_{a} \delta \\
& +s Y_{d}-\frac{m_{a}}{\gamma} \widetilde{\delta} \dot{\hat{\delta}}^{2} \frac{m_{a}}{\beta} \tilde{\theta} \dot{\hat{\theta}},
\end{aligned}
$$

where the unknown positive constant $\delta$ is defined as $\delta=$ $m_{a}^{-1}\left(\Delta^{*}+\varepsilon^{*}+d^{*}\right)$ and has been mentioned previously. Substituting (24) into (42) yields

$$
\begin{aligned}
\dot{V} & \leq s g(t)\left[-k s-\frac{\widehat{\theta} s}{2 a^{2}} \varphi^{T}(x) \varphi(x)-\widehat{\delta} \tanh \left(\frac{s}{v}\right)\right. \\
& \left.-\zeta Y_{d} \tanh \left(\frac{s Y_{d}}{v}\right)\right]+\frac{s^{2}\left\|W^{*}\right\|^{2}}{2 a^{2}} \varphi^{T}(x) \varphi(x)+\frac{a^{2}}{2} \\
& +|s| m_{a} \delta+s Y_{d}-\frac{m_{a}}{\gamma} \tilde{\delta} \dot{\delta}-\frac{m_{a}}{\beta} \tilde{\theta} \dot{\hat{\theta}}
\end{aligned}
$$

It should be noticed that $\widehat{\theta}>0$ and $\widehat{\delta}>0$ according to (25), (26), and Lemma 6. Therefore, it follows from (43) that

$$
\begin{aligned}
\dot{V} \leq & -k m_{a} s^{2}-\frac{m_{a} \theta s^{2}}{2 a^{2}} \varphi^{T}(x) \varphi(x) \\
& +\frac{s^{2}\left\|W^{*}\right\|^{2}}{2 a^{2}} \varphi^{T}(x) \varphi(x)+\frac{a^{2}}{2} \\
& +m_{a} \delta\left[|s|-s \tanh \left(\frac{s}{v}\right)\right] \\
& +\left[\left|s Y_{d}\right|-s Y_{d} \tanh \left(\frac{s Y_{d}}{v}\right)\right] \\
& -\frac{m_{a}}{\gamma} \widetilde{\delta}\left[\dot{\hat{\delta}}-\gamma s \tanh \left(\frac{s}{v}\right)\right] \\
& -\frac{m_{a}}{\beta} \widetilde{\theta}\left[\dot{\hat{\theta}}-\frac{\beta s^{2}}{2 a^{2}} \varphi^{T}(x) \varphi(x)\right] .
\end{aligned}
$$

Noting $\theta=m_{a}^{-1}\left\|W^{*}\right\|^{2}$ and substituting (25) and (26) into (42), we have

$$
\begin{aligned}
\dot{V} \leq & -k m_{a} s^{2}+m_{a} \delta\left[|s|-s \tanh \left(\frac{s}{v}\right)\right] \\
& +\left[\left|s Y_{d}\right|-s Y_{d} \tanh \left(\frac{s Y_{d}}{v}\right)\right]+\sigma_{1} m_{a} \tilde{\delta} \widehat{\delta} \\
& +\sigma_{2} m_{a} \tilde{\theta} \widehat{\theta}+\frac{a^{2}}{2} .
\end{aligned}
$$

By virtue of (7), we can further obtain

$$
\begin{aligned}
\dot{V} \leq & -k m_{a} s^{2}+0.2785 v\left(m_{a} \delta+1\right)+\sigma_{1} m_{a} \widetilde{\delta} \widehat{\delta} \\
& +\sigma_{2} m_{a} \widetilde{\theta} \widehat{\theta}+\frac{a^{2}}{2} .
\end{aligned}
$$

Using the inequalities

$$
\begin{aligned}
& \widetilde{\delta} \widehat{\delta}=\widetilde{\delta}(\delta-\widetilde{\delta}) \leq \frac{\delta^{2}}{2}-\frac{\widetilde{\delta}^{2}}{2}, \\
& \widetilde{\theta} \widehat{\theta}=\widetilde{\theta}(\theta-\widetilde{\theta}) \leq \frac{\theta^{2}}{2}-\frac{\tilde{\theta}^{2}}{2},
\end{aligned}
$$


we further have

$$
\begin{aligned}
\dot{V} \leq & -k m_{a} s^{2}-\frac{\sigma_{1} m_{a}}{2} \widetilde{\delta}^{2}-\frac{\sigma_{2} m_{a}}{2} \widetilde{\theta}^{2} \\
& +0.2785 v\left(m_{a} \delta+1\right)+m_{a}\left(\sigma_{1} \frac{\delta^{2}}{2}+\sigma_{2} \frac{\theta^{2}}{2}\right) \\
& +\frac{a^{2}}{2} \leq-\alpha V+\rho,
\end{aligned}
$$

where

$$
\begin{aligned}
& \alpha=\min \left\{2 k m_{a}, \sigma_{1} \gamma, \sigma_{2} \beta\right\}, \\
& \rho=0.2785 v\left(m_{a} \delta+1\right)+m_{a}\left(\sigma_{1} \frac{\delta^{2}}{2}+\sigma_{2} \frac{\theta^{2}}{2}\right)+\frac{a^{2}}{2} .
\end{aligned}
$$

Remark 14. From (49), we can know that $\rho$ can be made arbitrarily small by reducing the design parameters $v, \sigma_{1}, \sigma_{2}$, and $a$, while $\alpha$ can be made arbitrarily large by increasing the design parameters $k, \beta$, and $\gamma$. Thus, we can always have $\alpha \geq \rho / p$ by appropriately choosing the design parameters, and hence it follows from (48) that $\dot{V} \leq 0$ on $V=p$. Therefore, if $V(0) \leq p$, then $V(t) \leq p$ for all $t>0$. In other words, $\Pi_{1}$ is an invariant set [46] (please see [46] for details), and all the variables of $\Pi_{1}$ will stay in it as long as their initial conditions do be in $\Pi_{1}$. Note that we assume $V(0) \leq p$ in Theorem 11; therefore $V(t) \leq p$ for all $t>0$, and all the variables of $\Pi_{1}$ will stay in $\Pi_{1}$.

Multiplying (48) by $e^{\alpha t}$ yields

$$
(\dot{V}(t)+\alpha V(t)) e^{\alpha t} \leq \rho e^{\alpha t}
$$

which implies

$$
\frac{d}{d t}\left(V(t) e^{-\alpha t}\right) \leq \rho e^{-\alpha t}
$$

Integrating (51) over $[0, t]$, we have

$$
V(t) e^{\alpha t}-V(0) \leq \frac{1}{\alpha}\left(\rho e^{\alpha t}-\rho\right)
$$

then

$$
V(t) \leq(V(0)-\phi) e^{-\alpha t}+\phi
$$

where $\phi=\rho / \alpha$. From (53), it is known that $V, s, \widetilde{\delta}$, and $\widetilde{\theta}$ are bounded. Considering the definition of $V$ in (23) and applying (53), the following inequality hold:

$$
\frac{1}{2} s^{2} \leq V(t) \leq V(0)+\phi
$$

which implies that

$$
|s| \leq \sqrt{2(V(0)+\phi)} .
$$

Define $\omega=\left[e_{1}, e_{2}, \ldots, e_{n-1}\right]^{T}$. From (13), we know that there is a state-space representation for mapping $s=$ $\left[\Lambda^{T} 1\right] e_{1}$; that is, $\dot{\omega}=A_{s} \omega+b_{s} s$ with $\Lambda=\left[\lambda_{1}, \lambda_{2}, \ldots, \lambda_{n-1}\right]^{T}$, $b_{s}=[0,0, \ldots, 1]^{T}$, and $A_{s}$ being a stable matrix. It is known from this state-space representation that there is a positive constant $c_{0}$ such that $\left\|e^{A_{s} t}\right\| \leq c_{0} e^{-\lambda_{1} t}$ and the solution for $\omega$ is

$$
\omega(t)=e^{A_{s} t} \omega(0)+\int_{0}^{t} e^{A_{s}(t-\tau)} b_{s} s(\tau) d \tau .
$$

Accordingly, it follows that

$$
\|\omega(t)\| \leq c_{0}\|\omega(0)\| e^{-\lambda_{1} t}+c_{0} \int_{0}^{t} e^{-\lambda_{1}(t-\tau)}|s(\tau)| d \tau .
$$

Therefore, we have

$$
\|\omega(t)\| \leq c_{0}\|\omega(0)\|+\frac{c_{0} \sqrt{2(V(0)+\phi)}}{\lambda_{1}} .
$$

Noting $s=\Lambda^{T} \omega+e_{n}$ and $e=\left[\omega^{T}, e_{n}\right]^{T}$, we obtain

$$
\|e\| \leq\|\omega\|+\left|e_{n}\right| \leq(1+\|\Lambda\|)\|\omega\|+|s| .
$$

Substituting inequality (58) into the above inequality leads to

$$
\|e\| \leq \eta
$$

where $\eta=c_{0}(1+\|\Lambda\|)\|\omega(0)\|+\left[1+(1+\|\Lambda\|) c_{0} /\right.$ $\left.\lambda_{1}\right] \sqrt{2(V(0)+\phi)}$, whose size depends on the initial conditions and design parameters. Then property (i) of Theorem 11 holds.

In addition, according to (16) and (23), we have that $s^{2} / 2 \leq V$. Using (53), the following inequality holds:

$$
\lim _{t \rightarrow \infty}|s| \leq C
$$

where $C=\sqrt{2 \phi}$. It can be concluded form Remark 9 that $s$ and $e$ will eventually converge to compact sets $\Omega_{s}$ and $\Omega_{c}$ defined in Theorem 11. Note that the size of $\phi$ can be minimized by the design parameters $k, a, v, \sigma_{1}, \sigma_{2}, \gamma$, and $\beta$. Therefore, by appropriately online-tuning the design parameters, the compact sets $\Omega_{s}$ and $\Omega_{c}$ can be made as small as desired. This completes the proof.

Remark 15. In this paper, the main novelties lie on the following: firstly, in the light of the idea in the dynamic surface control [46], we find out the fact (30) is satisfied on the set $\Pi_{0} \times \Pi_{1}$. This fact is very useful to solve the difficulty of modeling the nonaffine function under semibounded condition. Secondly, we use a new method to model the nonaffine function as shown in (33) to (40), without using the mean value theorem. These are new works on nonaffine nonlinear systems.

Remark 16. The control performance can be improved in the sense of reducing the size of $\Omega_{s}$ by an appropriate choice of the design parameters $k, a, v, \sigma_{1}, \sigma_{2}, \gamma$, and $\beta$. Noting $\phi=\rho / \alpha$, it can be known from (61) that the decrease of $C$ can be achieved through increasing $\alpha$ and decreasing $\rho$. From Remark 14, it can be further known that increasing $k$, $\beta$, and $\gamma$ and decreasing $v, \sigma_{1}, \sigma_{2}$, and $a$ lead to the decrease of $C$ and result in the acceleration of the convergence rate of 
the varieties in the system. It is noted that if $\sigma_{1}$ and $\sigma_{2}$ are too small, it may not be enough to prevent the parameter estimates from drifting. If $k, \beta$, and $\gamma$ are big, the control energy is big. Therefore, in practical applications, the design parameters should be adjusted carefully to achieve suitable transient performance and control action.

\section{Simulation Results}

To illustrate the effectiveness and advantage of our proposed control scheme, two simulation examples are shown in this section.

Example 1. Consider a nonaffine nonlinear system described by

$$
\begin{aligned}
& \dot{x}_{1}=x_{2}, \\
& \dot{x}_{2}=f(x, v)+d(t), \\
& y=x_{1},
\end{aligned}
$$

where $f(x, u)=x_{1}^{2}+0.15 u^{3}+0.1\left(1+x_{2}^{2}\right) u+\sin (0.1 u)$ and $d(t)=0.5 \sin (2 t)$.

In accordance with (24), the controller in our scheme is designed as

$$
\begin{aligned}
u= & -3 s-\frac{\widehat{\theta} s}{2 a^{2}} \varphi^{T}(x) \varphi(x)-\widehat{\delta} \tanh \left(\frac{s}{0.15}\right) \\
& -Y_{d} \tanh \left(\frac{s Y_{d}}{0.15}\right)
\end{aligned}
$$

with the adaption laws as

$$
\begin{aligned}
& \dot{\hat{\delta}}=\gamma s \tanh \left(\frac{s}{0.15}\right)-\sigma_{1} \gamma \widehat{\delta}, \\
& \dot{\hat{\theta}}=\frac{\beta s^{2}}{2 a^{2}} \varphi^{T}(x) \varphi(x)-\sigma_{2} \beta \widehat{\theta},
\end{aligned}
$$

where $\sigma_{1}=0.2, \sigma_{2}=0.35, \lambda_{1}=3, \gamma=\beta=1$, and $a=0.25$. As for RBFNN, it is well known that the selection of the centers and widths would have a great influence on the performance of the designed controller in practice. According to [49], Gaussian RBFNN arranged on a regular lattice on $R^{n}$ can uniformly approximate sufficiently smooth functions on closed, bounded subsets. Accordingly, in our simulation studies, the centers and widths are chosen on a regular lattice in the respective compact sets. Particularly, neural network $W^{T} \varphi(x)$ in Example 1 contains 9 nodes with centers evenly spaced in the interval $[-0.5,0.5] \times[-1,1]$ and widths equal to 2 . The initial conditions are supposed to be $\left[x_{1}(0), x_{2}(0)\right]^{T}=[0.5,0]^{T}, \widehat{\theta}(0)=0$, and $\widehat{\delta}(0)=0$. The desired trajectory $y_{d}=0.5[\sin t+\sin (0.5 t)]$.

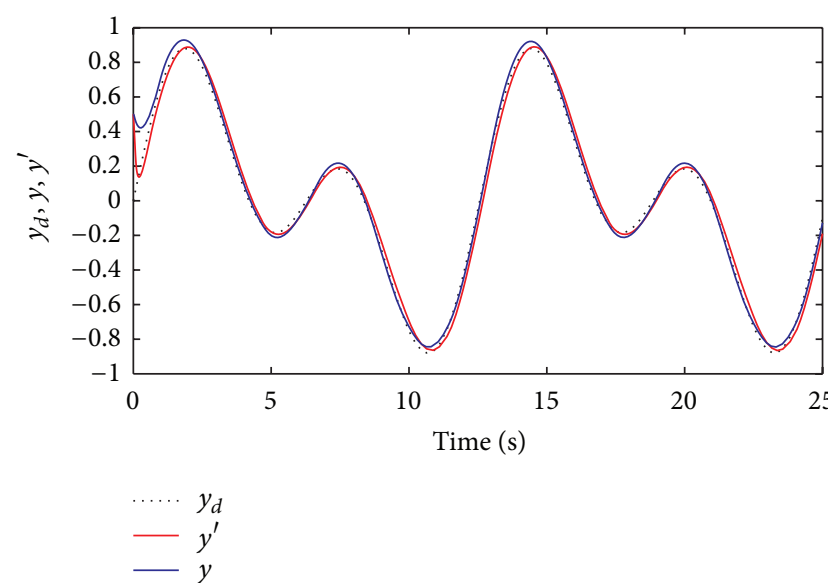

Figure 1: System outputs $y, y^{\prime}$ and desired trajectory $y_{d}$ in Example 1.

For comparison, the scheme in [47] is also applied to system (62). According to [47], the adaptive neural controller is designed as follows:

$$
\begin{aligned}
u & =-3 z_{2}-\frac{\widehat{\theta} z_{2}}{2 a^{2}} \varphi^{T}\left(Z_{2}\right) \varphi\left(Z_{2}\right)+x_{20} \\
z_{2} & =x_{2}-\alpha_{1} \\
\alpha_{1} & =-9 e_{1}+x_{10}
\end{aligned}
$$

where $a=0.25, x_{10}=0.5$, and $x_{20}=0$ in this simulation, and the details on the vector variable $Z_{2}$ and the adaption laws can be seen in [47]. The adaption gains for [47] are selected as the same as our scheme. The simulation results for both schemes are shown in Figures 1-4. In Figure 1, the black dotted line represents the desired trajectory $y_{d}$, the red line represents the system output $y^{\prime}$ for scheme in [47], and the blue line represents the system output $y$ for our scheme. It can be observed from Figure 1 that the system outputs of both schemes track the desired trajectory very well. Figure 2 shows the tracking errors of both schemes. In this figure, we can know that the tracking error of our scheme is smaller than [47] under the same conditions. Figure 3 shows the system states of both schemes. Figure 4 illustrates the control inputs of both schemes. Based on these simulation results, we can know that our scheme can not only guarantee the boundedness of all the signals but also achieve better tracking performance than [47].

Example 2. To further show the effectiveness of the proposed scheme, consider a one-link robot described as follows [44]:

$$
\begin{aligned}
& \dot{x}_{1}=x_{2}, \\
& \dot{x}_{2}=f(x, u)+d(t)
\end{aligned}
$$




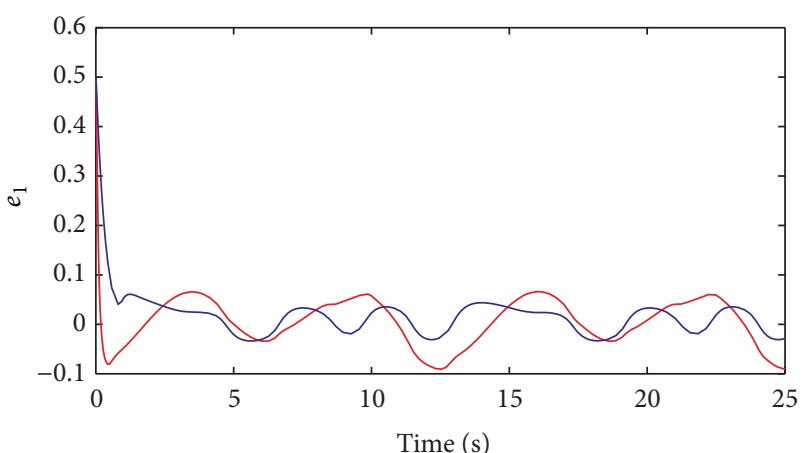

Scheme in [47]

Our scheme

FIGURE 2: Tracking error $e_{1}$ in Example 1.

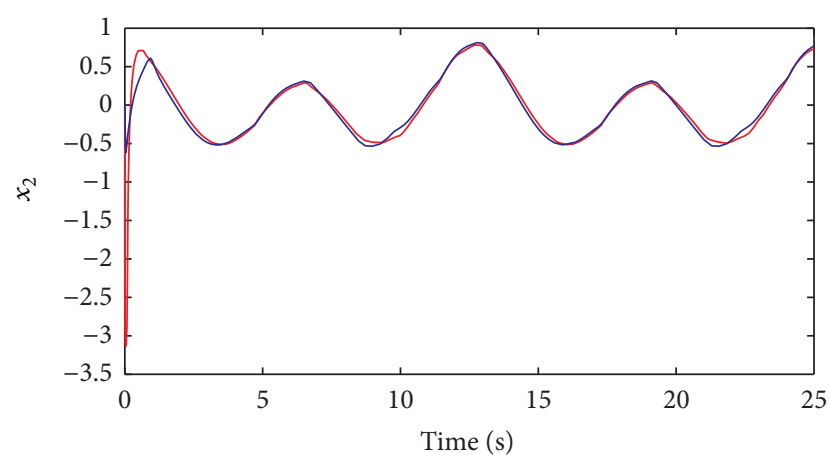

- Scheme in [47]

_ Our scheme

FIGURE 3: System state $x_{2}$ in Example 1.

with

$$
\begin{aligned}
& f(x, u)=-p_{1} x_{2}+p_{2} x_{2}^{2} \cos \left(x_{1}\right)-p_{3} \sin \left(x_{1}\right) \\
&+\psi(u)+|0.2 \sin (\psi(u))|, \\
& \psi(u)= \begin{cases}u-0.5, & u \geq 0.5, \\
0, & -0.5<u<0.5, \\
u+0.5 & u \leq-0.5,\end{cases} \\
& d(t)= 0.3 \sin (t),
\end{aligned}
$$

where system state $x_{1}$ denotes the angular displacement $\theta, x_{2}$ denotes its time derivative $\dot{\theta}$, and $d(t)$ denotes the external disturbance. More details on the description of model (66) can be seen in [44]. It can be seen that function $f(x, u)$ is indifferentiable with respect to $u$; therefore the scheme in [47] is unavailable. However, it is easily seen that Assumption 1 is satisfied, and our scheme is still applicable. For the purpose of

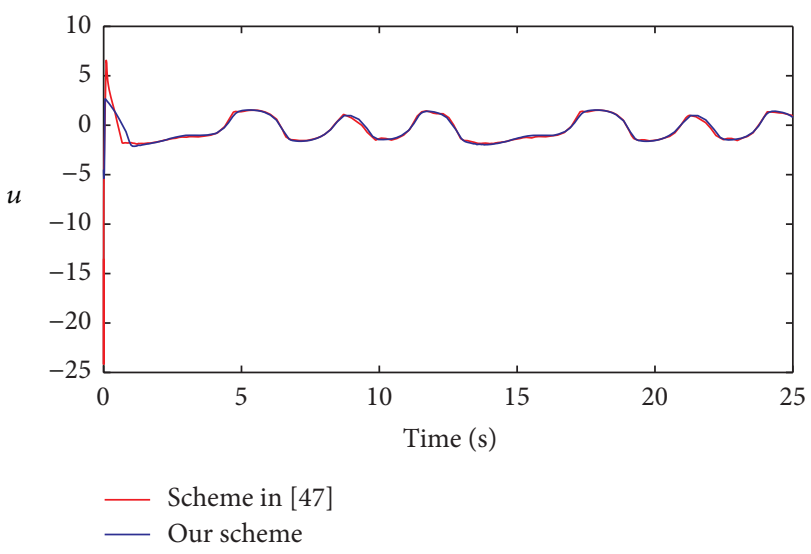

FIgURE 4: Control input $u$ in Example 1.

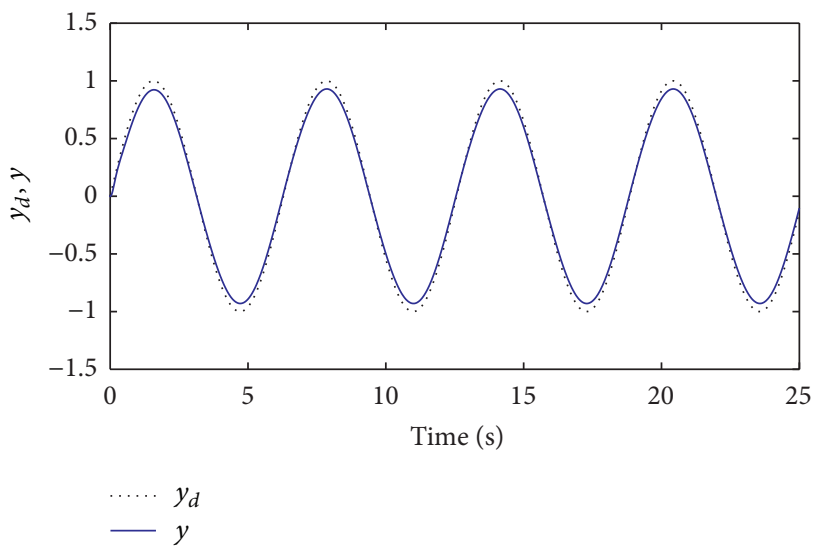

FIgURE 5: System output $y$ and desired trajectory $y_{d}$ in Example 2.

simulation, let $p_{1}=1, p_{2}=3.5$, and $p_{3}=35$. In accordance with (24), the adaptive neural controller is designed as

$$
\begin{aligned}
u= & -5 s-\frac{\widehat{\theta} s}{2 a^{2}} \varphi^{T}(x) \varphi(x)-\widehat{\delta} \tanh \left(\frac{s}{0.25}\right) \\
& -Y_{d} \tanh \left(\frac{s Y_{d}}{0.25}\right),
\end{aligned}
$$

with the adaption laws as

$$
\begin{aligned}
& \dot{\hat{\delta}}=\gamma s \tanh \left(\frac{s}{0.25}\right)-\sigma_{1} \gamma \widehat{\delta}, \\
& \dot{\hat{\theta}}=\frac{\beta s^{2}}{2 a^{2}} \varphi^{T}(x) \varphi(x)-\sigma_{2} \beta \widehat{\theta},
\end{aligned}
$$

where $\sigma_{1}=\sigma_{2}=0.45, \lambda_{1}=5, \gamma=\beta=1$, and $a=0.25$. The RBFNNs are designed the same as Example 1. Set the initial conditions as $\left[x_{1}(0), x_{2}(0)\right]^{T}=[0,0]^{T}, \widehat{\theta}(0)=0$, and $\widehat{\delta}(0)=0$. The desired trajectory $y_{d}=\sin t$. The simulation results are depicted in Figures 5-7. From these results, it is clearly seen that excellent performance is achieved. 


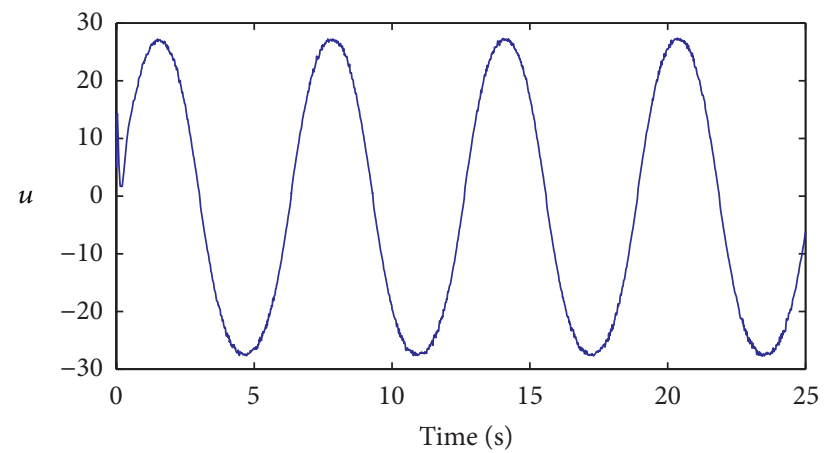

FIGURE 6: Control input $u$ in Example 2.
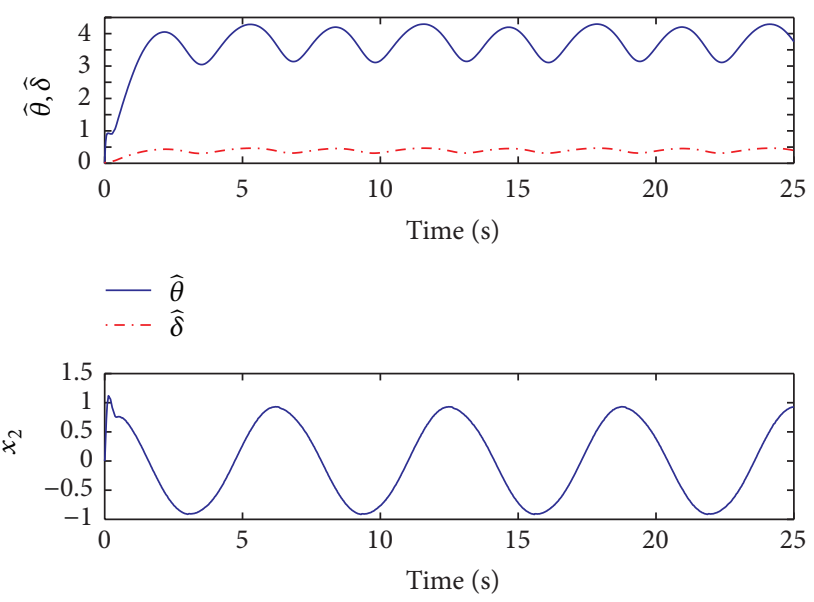

FIgUre 7: The curves of $\widehat{\theta}, \widehat{\delta}$, and $x_{2}$ in Example 2.

\section{Conclusion}

A novel adaptive neural control scheme has been presented for nonaffine nonlinear systems by modeling the nonaffine nonlinear function appropriately. Compared with the existing results, there is no knowledge of differential condition of nonaffine nonlinear function in this paper, and the assumption on the nonaffine nonlinear function is more relaxed as only semibounded condition is required. The stability of the closed-loop system has been proved using rigorous Lyapunov analysis and invariant set theory. Finally, simulation results have been shown to illustrate the effectiveness of the proposed adaptive control scheme. Moreover, it can be known from the previous analysis and simulation that the external disturbance of system is eliminated effectively by using robust compensator to suppress the undesirable inaccuracy, and excellent control performance is therefore achieved.

\section{Competing Interests}

The authors declare that they have no competing interests.

\section{References}

[1] J. Wang and J. Hu, "Robust adaptive neural control for a class of uncertain non-linear time-delay systems with unknown deadzone non-linearity," IET Control Theory \& Applications, vol. 5, no. 15, pp. 1782-1795, 2011.

[2] W. Chen and L. Jiao, "Adaptive tracking for periodically timevarying and nonlinearly parameterized systems using multilayer neural networks," IEEE Transactions on Neural Networks, vol. 21, no. 2, pp. 348-351, 2010.

[3] C.-F. Hsu, C.-M. Lin, and T.-T. Lee, "Wavelet adaptive backstepping control for a class of nonlinear systems," IEEE Transactions on Neural Networks, vol. 17, no. 5, pp. 1175-1183, 2006.

[4] T.-S. Li, D. Wang, G. Feng, and S.-C. Tong, "A DSC approach to robust adaptive NN tracking control for strict-feedback nonlinear systems," IEEE Transactions on Systems, Man, and Cybernetics, Part B: Cybernetics, vol. 40, no. 3, pp. 915-927, 2010.

[5] Z. Liu, X. Dong, J. Xue, and L. Zhang, "Adaptive tracking control for nonlinear systems with a class of input nonlinearities," Asian Journal of Control, vol. 18, no. 2, pp. 771-778, 2016.

[6] Y. Wen and X. Ren, "Neural networks-based adaptive control for nonlinear time-varying delays systems with unknown control direction," IEEE Transactions on Neural Networks, vol. 22, no. 10, pp. 1599-1612, 2011.

[7] S.-C. Tong, Y.-M. Li, G. Feng, and T.-S. Li, "Observer-based adaptive fuzzy backstepping dynamic surface control for a class of MIMO nonlinear systems," IEEE Transactions on Systems, Man, and Cybernetics, Part B: Cybernetics, vol. 41, no. 4, pp. 1124-1135, 2011.

[8] H. Lee, "Robust adaptive fuzzy control by backstepping for a class of MIMO nonlinear systems," IEEE Transactions on Fuzzy Systems, vol. 19, no. 2, pp. 265-275, 2011.

[9] S. Jagannathan and P. He, "Neural-network-based state feedback control of a nonlinear discrete-time system in nonstrict feedback form," IEEE Transactions on Neural Networks, vol. 19, no. 12, pp. 2073-2087, 2008.

[10] Q. Zhou, P. Shi, J. Lu, and S. Xu, "Adaptive output-feedback fuzzy tracking control for a class of nonlinear systems," IEEE Transactions on Fuzzy Systems, vol. 19, no. 5, pp. 972-982, 2011.

[11] T.-P. Zhang, H. Wen, and Q. Zhu, "Adaptive fuzzy control of nonlinear systems in pure feedback form based on input-tostate stability," IEEE Transactions on Fuzzy Systems, vol. 18, no. 1, pp. 80-93, 2010.

[12] M. Chen, S. S. Ge, and B. V. E. How, "Robust adaptive neural network control for a class of uncertain MIMO nonlinear systems with input nonlinearities," IEEE Transactions on Neural Networks, vol. 21, no. 5, pp. 796-812, 2010.

[13] T. Zhang, S. S. Ge, and C. C. Hang, "Adaptive neural network control for strict-feedback nonlinear systems using backstepping design," Automatica, vol. 36, no. 12, pp. 1835-1846, 2000.

[14] S. C. Tong, Y. Li, Y. M. Li, and Y. J. Liu, "Observer-based adaptive fuzzy backstepping control for a class of stochastic nonlinear strict-feedback systems," IEEE Transactions on Systems, Man, and Cybernetics B, vol. 41, no. 6, pp. 1693-1704, 2011.

[15] B. Chen, X. Liu, K. Liu, and C. Lin, "Fuzzy-approximationbased adaptive control of strict-feedback nonlinear systems with time delays," IEEE Transactions on Fuzzy Systems, vol. 18, no. 5, pp. 883-892, 2010.

[16] T.-S. Li, D. Wang, G. Feng, and S.-C. Tong, "A DSC approach to robust adaptive nn tracking control for strict-feedback nonlinear systems," IEEE Transactions on Systems, Man, and Cybernetics, Part B: Cybernetics, vol. 40, no. 3, pp. 915-927, 2010. 
[17] M. Krstic, I. Kanellakopoulos, and P. Kokotovic, Nonlinear and Adaptive Control Design, Wiley, New York, NY, USA, 1995.

[18] X. Dong, G. Chen, and L. Chen, "Adaptive control of the uncertain duffing oscillator," International Journal of Bifurcation and Chaos in Applied Sciences and Engineering, vol. 7, no. 7, pp. 1651-1658, 1997.

[19] L. R. Hunt and G. Meyer, "Stable inversion for nonlinear systems," Automatica, vol. 33, no. 8, pp. 1549-1554, 1997.

[20] A. Ferrara and L. Giacomini, "Control of a class of mechanical systems with uncertainties via a constructive adaptive/second order VSC approach," Journal of Dynamic Systems, Measurement, and Control, vol. 122, no. 1, pp. 33-39, 2000.

[21] C. Wang, D. J. Hill, S. S. Ge, and G. Chen, "An ISS-modular approach for adaptive neural control of pure-feedback systems," Automatica, vol. 42, no. 5, pp. 723-731, 2006.

[22] J.-H. Park, S.-H. Huh, S.-H. Kim, S.-J. Seo, and G.-T. Park, "Direct adaptive controller for nonaffine nonlinear systems using self-structuring neural networks," IEEE Transactions on Neural Networks, vol. 16, no. 2, pp. 414-422, 2005.

[23] J.-H. Park, G.-T. Park, S.-H. Kim, and C.-J. Moon, "Direct adaptive self-structuring fuzzy controller for nonaffine nonlinear system," Fuzzy Sets and Systems, vol. 153, no. 3, pp. 429-445, 2005.

[24] Y.-G. Leu, W.-Y. Wang, and T.-T. Lee, "Observer-based direct adaptive fuzzy-neural control for nonaffine nonlinear systems," IEEE Transactions on Neural Networks, vol. 16, no. 4, pp. 853861, 2005.

[25] S. S. Ge, C. C. Hang, and T. Zhang, "Adaptive neural network control of nonlinear systems by state and output feedback," IEEE Transactions on Systems, Man, and Cybernetics, Part B: Cybernetics, vol. 29, no. 6, pp. 818-828, 1999.

[26] M. Chen and S. S. Ge, "Direct adaptive neural control for a class of uncertain nonaffine nonlinear systems based on disturbance observer," IEEE Transactions on Cybernetics, vol. 43, no. 4, pp. 1213-1225, 2013.

[27] S.-L. Dai, C. Wang, and M. Wang, "Dynamic learning from adaptive neural network control of a class of nonaffine nonlinear systems," IEEE Transactions on Neural Networks and Learning Systems, vol. 25, no. 1, pp. 111-123, 2014.

[28] W. J. Dong, Y. Y. Zhao, Y. M. Chen, and J. A. Farrell, "Tracking control for nonaffine systems: a self-organizing approximation approach," IEEE Transactions on Neural Networks and Learning Systems, vol. 23, no. 2, pp. 223-235, 2012.

[29] S. C. Tong and Y. M. Li, "Adaptive fuzzy output feedback backstepping control of pure-feedback nonlinear systems via dynamic surface control technique," International Journal of Adaptive Control and Signal Processing, vol. 27, no. 7, pp. 541561, 2013.

[30] Y. M. Li and S. C. Tong, "Adaptive fuzzy output-feedback control of pure-feedback uncertain nonlinear systems with unknown dead zone," IEEE Transactions on Fuzzy Systems, vol. 22, no. 5, pp. 1341-1347, 2014.

[31] W. D. Zhou, C. Y. Liao, L. Zheng, and M. M. Liu, "Adaptive fuzzy control for a class of chaotic systems with nonaffine inputs," Nonlinear Dynamics, vol. 79, no. 4, pp. 2609-2621, 2014.

[32] Y.-S. Huang, D.-Q. Zhou, and X. X. Chen, "Decentralized direct adaptive output feedback fuzzy $\mathbf{H}_{\mathbf{\infty}}$ tracking design of largescale nonaffine nonlinear systems," Nonlinear Dynamics, vol. 58, no. 1, pp. 153-167, 2009.

[33] J. Xiang, Y. Li, and W. Wei, "Stabilisation of a class of non-affine systems via modelling error compensation," IET Control Theory \& Applications, vol. 2, no. 2, pp. 108-116, 2008.
[34] J.-H. Park and S.-H. Kim, "Direct adaptive output-feedback fuzzy controller for a nonaffine nonlinear system," IET Control Theory \& Applications, vol. 151, no. 1, pp. 65-72, 2004.

[35] A. Boulkroune, M. M'Saad, and M. Farza, "Fuzzy approximation-based indirect adaptive controller for multiinput multi-output non-affine systems with unknown control direction," IET Control Theory \& Applications, vol. 6, no. 17, pp. 2619-2629, 2012.

[36] J.-H. Park and G.-T. Park, "Robust adaptive fuzzy controller for non-affine nonlinear systems with dynamic rule activation," International Journal of Robust and Nonlinear Control, vol. 13, no. 2, pp. 117-139, 2003.

[37] A. Boulkroune, M. Tadjine, M. M'Saad, and M. Farza, "Adaptive fuzzy controller for non-affine systems with zero dynamics," International Journal of Systems Science, vol. 40, no. 4, pp. 367382, 2009.

[38] S. Labiod and T. M. Guerra, "Adaptive fuzzy control of a class of SISO nonaffine nonlinear systems," Fuzzy Sets and Systems, vol. 158, no. 10, pp. 1126-1137, 2007.

[39] A. Boulkroune, M. Tadjine, M. M'Saad, and M. Farza, "Adaptive control based on SPO for nonaffine nonlinear systems and its application to CSTR system," Mediterranean Journal of Measurement and Control, vol. 3, no. 2, pp. 93-103, 2007.

[40] T. Zhang, "Adaptive control of first-order nonlinear systems with input non-affine nonlinearities," in Proceedings of the 45th IEEE Conference on Decision and Control, pp. 3436-3441, IEEE, San Diego, Calif, USA, 2006.

[41] T. P. Zhang and S. S. Ge, "Adaptive neural network tracking control of MIMO nonlinear systems with unknown dead zones and control directions," IEEE Transactions on Neural Networks, vol. 20, no. 3, pp. 483-497, 2009.

[42] J. Zhou, C. Zhang, and C. Wen, "Robust adaptive output control of uncertain nonlinear plants with unknown backlash nonlinearity," IEEE Transactions on Automatic Control, vol. 52, no. 3, pp. 503-509, 2007.

[43] X.-S. Wang, C.-Y. Su, and H. Hong, "Robust adaptive control of a class of nonlinear systems with unknown dead-zone," Automatica, vol. 40, no. 3, pp. 407-413, 2004.

[44] M. L. Corradini and G. Orlando, "Robust stabilization of nonlinear uncertain plants with backlash or dead zone in the actuator," IEEE Transactions on Control Systems Technology, vol. 10, no. 1, pp. 158-166, 2002.

[45] Z. C. Liu, X. M. Dong, J. P. Xue, and Y. Chen, "Adaptive neural control for a class of time-delay systems in the presence of backlash or dead-zone non-linearity," IET Control Theory \& Applications, vol. 8, no. 11, pp. 1009-1022, 2014.

[46] D. Swaroop, J. K. Hedrick, P. P. Yip, and J. J. Gerdes, "Dynamic surface control for a class of nonlinear systems," IEEE Transactions on Automatic Control, vol. 45, no. 10, pp. 1893-1899, 2000.

[47] M. Wang, S. S. Ge, and K.-S. Hong, "Approximation-based adaptive tracking control of pure-feedback nonlinear systems with multiple unknown time-varying delays," IEEE Transactions on Neural Networks, vol. 21, no. 11, pp. 1804-1816, 2010.

[48] T. Zhang, S. S. Ge, and C. C. Hang, "Stable adaptive control for a class of nonlinear systems using a modified Lyapunov function," IEEE Transactions on Automatic Control, vol. 45, no. 1, pp. 129132, 2000.

[49] R. M. Sanner and J.-J. E. Slotine, "Gaussian networks for direct adaptive control," IEEE Transactions on Neural Networks, vol. 3, no. 6, pp. 837-863, 1992. 


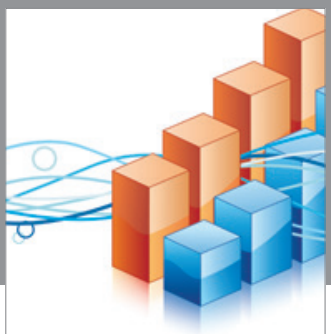

Advances in

Operations Research

vatem alat4

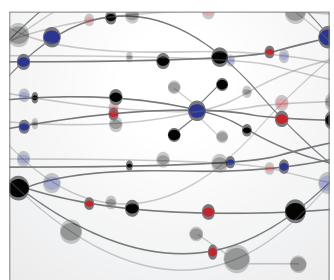

\section{The Scientific} World Journal
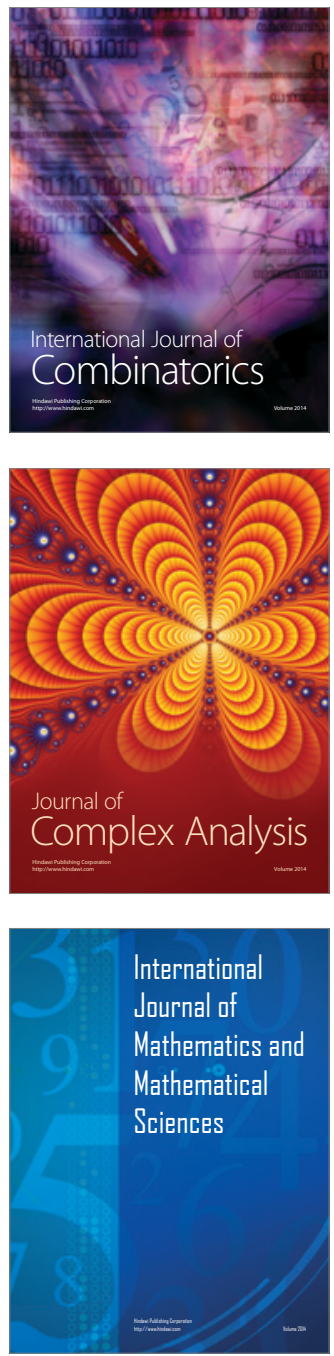
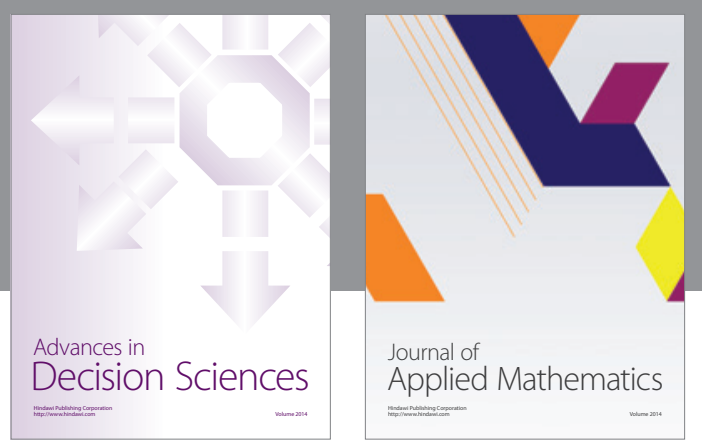

Algebra

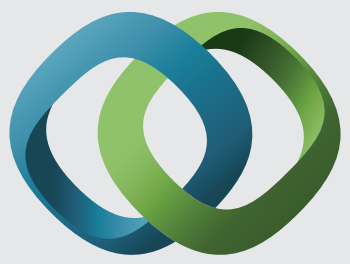

\section{Hindawi}

Submit your manuscripts at

http://www.hindawi.com
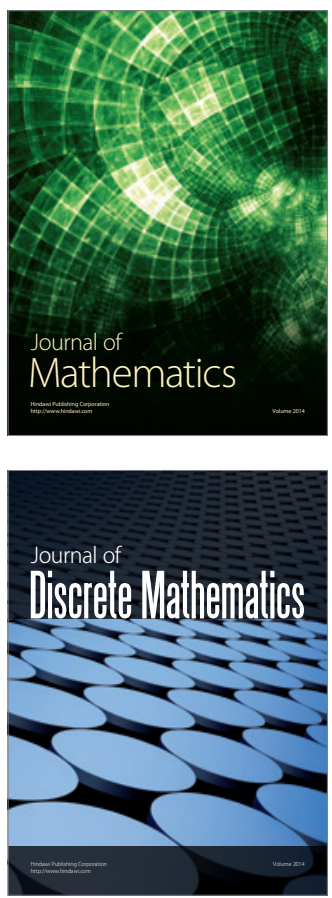

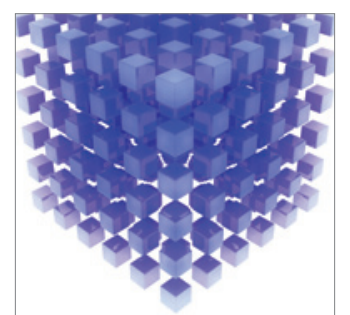

Mathematical Problems in Engineering
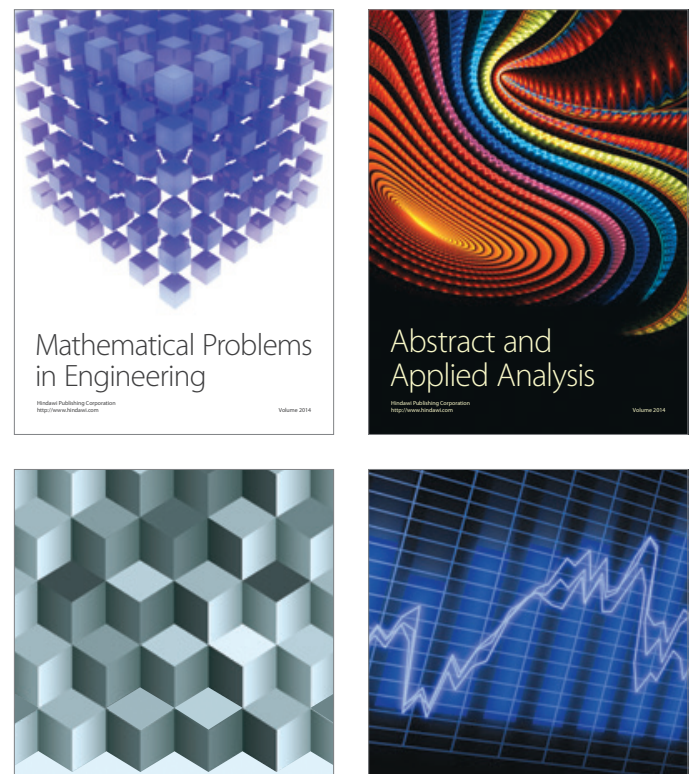

Journal of

Function Spaces

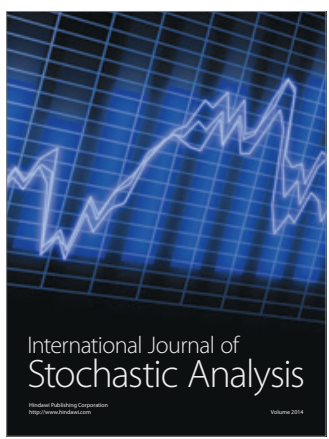

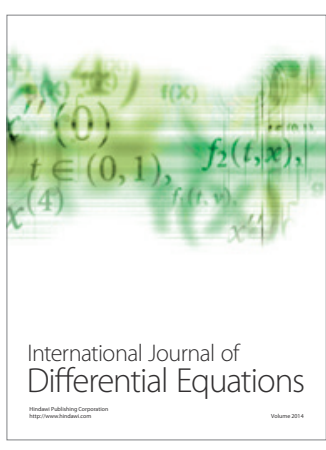
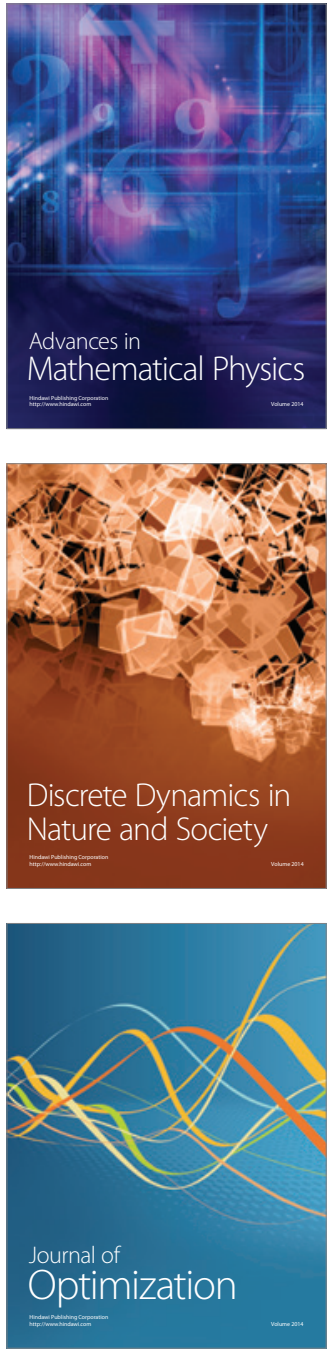